\title{
Human butyrylcholinesterase knock-out equivalent: Potential to assess role in Alzheimer's disease
}

\author{
Gumpeny Ramachandra Sridhar ${ }^{*}$, Talluri Sekhar ${ }^{2}$, Padmanabhuni Venkata Nageswara Rao ${ }^{3}$, \\ Allam Appa Rao ${ }^{4}$ \\ ${ }^{1}$ Endocrine and Diabetes Center, Visakhapatnam, India; ${ }^{2}$ Corresponding Author: sridharvizag@gmail.com \\ ${ }^{2}$ Department of Biotechnology, GITAM University, Visakhapatnam, India \\ ${ }^{3}$ Department of Computer Science, GITAM University, Visakhapatnam, India \\ ${ }^{4}$ C. R. Rao Advanced Institute for Mathematics, Statistics \& Computer Science (AIMSCS), Hyderabad, India
}

Received 5 April 2012; revised 22 May 2012; accepted 10 June 2012

\section{ABSTRACT}

Butyrylcholinesterase (BChE) is an enzyme which has been shown to be involved in the pathogenesis, treatment and prognosis of Alzheimer's disease. In its current form, however, evidence is equivocal with all of the associations. Variant forms of the protein exist, where the enzymatic function is lost to varying degrees. We performed in silico evaluation of these variants. Bioinformatics and molecular modeling, based on data from ESTHER database and Protein Data Bank (RCSB), were used for in silico predictions of the structures of the silent variants that involve a single amino acid change. Variants with loss of enzyme activity were evaluated for structural changes near the active site and the thermodynamic stability of the variants was estimated. The results indicated that the loss of activity of the variants can, in most cases, be attributed to structural changes in the active site or to lower thermodynamic stability. Our results showed that the loss of enzyme activity may be due to changes in the active site, oligomerization or loss of structural stability. Individuals with loss of function mutation of BChE can be studied and followed up for their proneness or resistance to cognitive decline with aging.

Keywords: Apnea; Anesthesia; Insulin Resistance; Mutants; Thermal Stability

\section{INTRODUCTION}

The cholinergic deficit hypothesis and the formation of amyloid plaques containing beta-amyloid peptide related to Alzheimer's disease have as a common pathogenic factor, cholinesterase enzymes that belong to the family of serine hydrolases [1,2]. Butyrylcholinesterase is produced by the liver and is secreted into the circulation, unlike acetylcholinesterase (AChE), the other member of the enzyme family. A tetrameric glycoprotein (mol mass $342 \mathrm{kDa}$ ), BChE is also seen in the intestinal mucosa and white matter of central nervous system [3]. The enzyme consists of four identical subunits, each with an active site. Although the physiological role of $\mathrm{BChE}$ is unclear, it serves as a marker of exposure to organophosphate pesticides and is also involved in metabolizing drugs such as cocaine, heroin and muscle relaxants such as suxamethonium [3].

$\mathrm{AChE}$ and $\mathrm{BChE}$ belong to a family of serine hydroxylases, having sequence homology with membrane associated proteins such as thyroglobulin and glutactin [4]. The rank ordering of serine and histidine positions within the linear sequences suggests that cholinesterase family evolved from convergent evolution. There is a $50 \%-52 \%$ residue identity between $\mathrm{AChE}$ and $\mathrm{BChE}$ [4]. The relationship between the structure and function of $\mathrm{AChE}$ has been studied experimentally as well as computationally [5]; this knowledge is helpful in elucidating the relationship between structure and function of $\mathrm{BChE}$ [6]. A number of variant BChEs have been identified, including mutations of deletion, missense and nonsense varieties. More than one kind of mutation can also occur [7-9]. BChE is coded by the BChE gene (3q26.1-q26.2) which has four exons [10]. Other forms of BCHE variants may result in the interaction of products from BCHE and AChE (2q33-q35) genes. Variant forms of the enzyme are seen in particular ethnic groups such as individuals from Vysya community of India [11].

Studies on the relation between BChE and Alzheimer's disease have been done in terms of etiopathogenesis, predisposition and drug therapy. Altered levels of AChE and $\mathrm{BChE}$ were reported in the cerebral cortex of individuals with Alzheimer's disease [12]. When the AChE activity in human plasma was assessed in subjects with 
Alzheimer's disease, the level was lower compared to $\mathrm{BChE}$; however the levels and patterns of the molecular forms were similar to those in individuals with silent BChE forms [13]. Darvesh et al. reported that although $\mathrm{BChE}$ is pathogenically related to lesions of Alzheimer's disease, the molecular forms of the enzyme from the brain of affected individuals are not modified in terms of their primary structure, implying that atypical histochemical behaviour may result from interaction of the protein with other molecules in the cortical lesions [14]. The ability of alanine-to-threonine substitutions and amyloid diseases as applied to $\mathrm{BChE}$ was shown to be compatible with inverse preferences of alanine to form helices and of threonine to support beta-sheets [15]. Despite being protective at an earlier age, amyloid formation sets in later in life. In view of a large pool of BChE being found in the glia located in the deeper cortical and subcortical structures, it is possible that it may be a potential therapeutic target for subcortical pathological processes [16].

An interaction of $\mathrm{BChE}$ with apoliprotein $\mathrm{E}$ (ApoE) appears to underlie the pathogenesis of Alzheimer's disease. In a recent study that investigated the interrelation between $\mathrm{ApoE}$ and $\mathrm{BChE}$ levels, levels of both strongly correlated with CMRglc, cerebral A beta load and CSF P-tau $(r=0.73, p<0.0001, n=33)$. High ApoE was associated with increased pool of dormant $\mathrm{BChE}$ molecules having high intrinsic catalytic rate in cerebrospinal fluid, suggesting that high ApoE levels cause changes involving cholinergic deficits by disturbing cholinergic activity dependent activity of neurons and glial cells [17]. Based on a retrospective analysis of data, a continuum was defined by gender and genotype. In early Alzheimer's disease men who were carriers of BChE-K variants with ApoE4 alleles had medial temporal atrophy, synaptic failure and cognitive decline [18]. Whereas carriers of BChE-K and ApoE4 stand an increased risk of Alzheimer's disease, those who do not carry APOE4 appear to be protected, in those with BCHE-K. The interrelationship was hypothesized to arise through a differential phenotypic modulation of $\mathrm{BChE}$ by ApoE4, which forms an interesting avenue for future studies [19]. High ApoE was associated with reduced amounts of $\mathrm{BChE}$ as well as differentially related to levels of cytokine Il-1B, a proinflammatory cytokine [20]. The interaction may occur through central modulation of cholinergic activity and A beta load in the brain. A post-hoc exploratory analysis of rivastigmine in the InDDEx study showed that progresssion to Alzheimer's disease and hippocampal loss was highest in those with both APOE4 and BChE-K alleles [21]. Presence of hyperhomocysteinemia was associated with more rapid decline in cognitive function associated with BChE-K [22]. Similarly BChE has been shown to interact with 14-3-3 zeta in influencing the risk of de- veloping Alzheimer's disease [23]. In addition, BChE has been proposed to be a link between the well recognized association between Alzheimer's disease and type 2 diabetes mellitus [24,25]. Variant forms of BChE have been reported from different geographic regions of the world, including India [26,27]. However association between $\mathrm{BChE}$ variants and propensity to Alzheimer's disease has shown ethnic differences $[28,29]$.

The active site of $\mathrm{BChE}$ is formed by catalytic triad amino acids Ser198-His438-Glu325 [30]. It is located at the bottom of a $20 \mathrm{~A}^{\circ}$ long narrow gorge, and catalyses hydrolysis in two steps: acylation followed by release of choline molecule and next deacylation of acyl-enzyme formed in the first step [7]. Other functional regions in the active site gorge have been identified: an oxy-anion hole (Gly116, Gly117 and Ala199), a site that stabilizes binding based on $\pi$-cation interactions (Trp82) and an acyl binding pocket consisting of a hydrophobic patch of residues (Leu286, Val288) [6,31,32]. Some of the residues such as Asp70 in the peripheral anionic site (Tyr332, Asp70) at the entrance of the active site gorge are predicted to have substantial mobility due to the presence of the flexible omega loop near the entrance of the active site gorge.

Asanuma et al. carried out a structural analysis of the L330I, G365R, and R515C mutations of human BChE [33]. The amino acid substitution at L330I was adjacent to hydrophobic residues that form the channel domain of the active center of the model. This side chain faced the side opposite the active center. They found that the amino acid substitution at G365R was located at the position most remote from the active center, and that this substitution site was exposed to the surface of the BChE protein. They also reported that the guanidyl residue of native Arg 515 was hydrogen-bonded to the carboxyl group of Asp 395 in an alpha helix adjacent to the active site. They concluded that point mutations may cause steric effects on $\mathrm{hBChE}$ activity. However, these observations were only deduced from a published model of the computer-generated three-dimensional structure of $\mathrm{hBChE}$ (based on the structure of acetylcholinesterase). The structures of the native form of human $\mathrm{BChE}$ and $\mathrm{BChE}$ bound to several substrate analogs are now available. The structural and thermodynamic analysis reported here are based on the experimentally determined structures of $\mathrm{BChE}$ and are expected to be more reliable and informative than the earlier structural analyses of native variants which were performed before the availability of experimentally determined structures of BChE.

Manoharan et al. have investigated the effect of the L307P mutation in $\mathrm{BChE}$ using predictions of thermal stability and concluded that the loss of activity in the L307P variant could be attributed to structural destabilization [11]. The work reported here is a comprehensive 
analysis of all single residue variants of BChE that lead to complete or nearly complete loss of activity of BChE. In order to study the loss of function of BChE variants, we assessed their structural changes in the active site and thermodynamic stability.

\section{METHODS}

\subsection{ESTHER Database}

The ESTHER database for esterases [34], contains information on 70 natural mutants of $\mathrm{BChE}$. Out of the 70 natural variants of $\mathrm{BChE}, 51$ involve single amino acid substitution, eight involve truncation and six involve frameshifts. Out of the 51 variants involving amino acid substitutions, 25 variants, that produce a silent phenotype, were chosen for this study.

\subsection{Multiple Sequence Alignment}

The BChE enzyme sequences of different organisms available in the ESTHER database were aligned using ClustalW [35,36]. The multiple sequence alignment obtained was displayed through MView. This alignment was used to identify residues that are conserved. Residues were classified as conserved if identical or similar residues were present in $70 \%$ or more of the sequences studied.

\subsection{Protein Data Bank}

Structural information was obtained from the Protein Data Bank (www.rcsb.org) [37, 38]. The coordinates of atoms of recombinant human BChE (2PM8) [32], and the atomic coordinates of $\mathrm{BChE}$ in complex with a choline molecule $(1 \mathrm{P} 0 \mathrm{M})$ were used for structural analysis of native $\mathrm{BChE}$ [31]. In addition to these, 1P0I and 3DJY were also used for comparative modeling. 1P0I was chosen because it is the highest resolution structure for human BChE without substrate [31]. 2PM8 was chosen because the structure was obtained using the full length chain of human BChE. 1P0M was chosen because it includes a small substrate, butyrylcholine, which is similar to succinyl choline which is commonly used as a substrate to assess the BChE activity of human serum. 3DJY was used because it is the highest resolution structure with a bound substrate analog [39].

\subsection{Distance Based Cluster Analysis}

A dedicated PERL program was used to calculate all pair wise distances between the $\mathrm{Ca}$ atoms of residues that are implicated in variants that produce the silent phenotype. The distance matrix produced by this program was used as input for distance based clustering using UPGMA implemented in the PHYLIP package [40,41].
Njplot [42] was used for displaying the resulting trees.

\subsection{Molecular Surface, Surface Accessible Pockets and Internal Cavities}

The molecular surface and the cavities within BChE were identified and displayed using the molecular graphics program Pymol [43]. In addition, the CASTp server was used to obtain additional information regarding the accessible pockets and internal cavities. CASTp computes molecular surfaces and volumes and defines the cavities by using weighted Delaunay triangulation and the theory of alpha shapes $[44,45]$.

\subsection{Prediction of DDG}

The effect of amino acid substitutions on the thermal stability of BChE was evaluated by using two methods: I-Mutant2.0 and Popmusic2.0. Popmusic2.0 uses statistical potentials based on property encoding for prediction of thermal stability with an estimated rms error of 1.15 $\mathrm{kcal} / \mathrm{mol}$ [46]. I-Mutant2.0 is a support vector machine based tool that can be used for the prediction of the change in free energy of unfolding upon mutation. The estimated standard errors for prediction of thermal stability with I-Mutant2.0 are $1.30 \mathrm{kcal} / \mathrm{mol}$ [47].

\subsection{Prediction of Structural Perturbations at the Active Site}

The effect of amino acid substitution on the geometry of the active site was evaluated by constructing structural models of the silent variants by using the Modeller package of programs (Modeller 9v8). Modeller constructs structural models of the sequence of interest by maximizing a molecular probability density function [48]. The molecular probability density function includes terms for similarity to the templates and terms based on potentials of mean force which were derived from observed distributions of structural parameters in proteins [49]. Four experimentally determined structures of human $\mathrm{BChE}$ (1P0I, 2PM8, 1P0M and 3DJY), obtained from the Protein Databank [37,38] were used as templates for the structural modeling of the variants.

The structural models of the variants were further optimized by using an initial round of conjugate gradients minimization (maximum of 20 steps), followed by molecular dynamics optimization (maximum of 50 steps, initial temperature $300 \mathrm{~K}$ ) and a final round of conjugate gradients minimization (maximum of 20 steps). The structural differences of functional interest, between the native enzyme and the models of the variants were assessed quantitatively by comparing the distance matrices of residues that play a critical role in the activity of BChE. Distance matrices, involving all pairwise dis- 
tances between the CA atoms of the active site residues (Ser198, His438, Glu325) and the residues comprising the oxy-anion hole (Gly116, Gly117, Ala199) were computed for the native protein as well as for all the variants. The root-mean-square distance between the distance matrices of native and variant structures, dRMS, was computed as follows:

$$
\mathrm{dRMS}=\operatorname{sqrt}\left(\left(\sum\left(\mathrm{d}_{i j, \text { native }}-\mathrm{d}_{i j \text {,variant }}\right)^{2}\right) / \text { npairs }\right)
$$

where, $\mathrm{d}_{i j \text {,native }}$ is the distance between a pair of atoms $i$ and $j$, and $\mathrm{d}_{i j \text {,variant }}$ is the distance between the same pair of atoms in the structure of the variant; npairs is the total number of atom pairs in the distance matrix.

\section{RESULTS}

Comparative sequence analysis of silent variants:

Multiple sequence alignment of $\mathrm{BChE}$ sequences from 25 species available at the ESTHER database was used to check the degree of conservation of residues whose mutations produce silent phenotypes. We observed that the following residues exhibit 100\% conservation: G115, L125, Y128, D170, S198, A199. However, the following residues are not conserved: T250, E255, A328, R515, Q518.

\subsection{Structural Analysis of Silent Variants}

CASTp was used to identify the major cavities and the contributing residues in the experimentally determined structure of the full-length recombinant hBChE (2pm8) and in the complex of $\mathrm{BChE}$ with butyrylthiocholine $(1 \mathrm{p} 0 \mathrm{~m})$. The active site residues of $\mathrm{hBChE}$ are at the bottom of a deep pocket having a volume of $1643 \mathrm{~A}^{3}$ in the structure of the complex of hBChE $(1 \mathrm{p} 0 \mathrm{~m})$; this volume is intermediate between the volume of the same pocket in the two chains $\mathrm{A}$ and $\mathrm{B}$ of the full-length recombinant human butyrylcholinesterase (2pm8), 1725.7 $\mathrm{A}^{3}$ and $1602.5 \mathrm{~A}^{3}$ respectively. The following residues are observed on the surface of this pocket (pocket ID 77): 66-72, 79, 82-84, 115-117, 119-121, 125, 148, 149, $197-199,231,273,276,277,280,283-289,328-329,332$, $398,430,437-440,442$. The set of residues that line the corresponding pocket in the full-length recombinant $\mathrm{hBChE}$ are almost the same, with the following minor changes: residues $122,128,151,434$ are extra in the $\mathrm{A}$ chain pocket; $114,128,331,434$ are extra and 66, 199, $273,280,283,442$ are not part of the B chain pocket. The substantial differences in the volumes of the active site gorge, in chemically identical chains present in the same crystal, indicates a substantial flexibility in this region (Table 1).

An examination of the experimentally determined structure of full length hBChE shows that most of the residues whose mutation leads to silent phenotypes for BChE activity are on the surface of the central cavity that defines the active site (shown in Figure 1). Mutation of these residues would be expected to result in altered substrate binding; this explains the observed loss of activity for these residues. However, it is also apparent that all the residues whose mutations produce silent phenotypes are not spatially proximal. Therefore, a distance based cluster analysis was used to identify sets of residues that are close to each other.

\subsection{Distance Based Cluster Analysis of BChE Silent Variants}

Distance based clustering was used to identify residues that are near the binding site or near residues that define the binding site. Distance based cluster analysis (Figure 2) reveals that most of the residues whose mutations lead to silent phenotype are close to each other as revealed by the cluster of residues that includes Ser198 in Figure 2:

Table 1. Areas and volumes of major cavities in human butyrylcholinesterase.

\begin{tabular}{ccclc}
\hline ID & Area & Volume & Comments & PDBID \\
\hline 153 & 5146.1 & 11797.0 & Interface of chains A and B & $2 \mathrm{pm} 8$ \\
152 & 1269.8 & 3040.6 & Cavity in chain B (equivalent to 151 of chain A) & $2 \mathrm{pm} 8$ \\
151 & 1172.9 & 2485.3 & Cavity in chain A (equivalent to 152 of chain B) & $2 \mathrm{pm} 8$ \\
150 & 1061.1 & 1725.7 & Active site gorge in chain A & $2 \mathrm{pm} 8$ \\
149 & 925.7 & 1602.5 & Active site gorge in chain B & $2 \mathrm{pm} 8$ \\
78 & 901.0 & 1752.1 & Pocket which is interface in dimer & $1 \mathrm{p} 0 \mathrm{~m}$ \\
77 & 997.6 & 1643.1 & Gorge containing active site & $1 \mathrm{p} 0 \mathrm{~m}$ \\
\hline
\end{tabular}




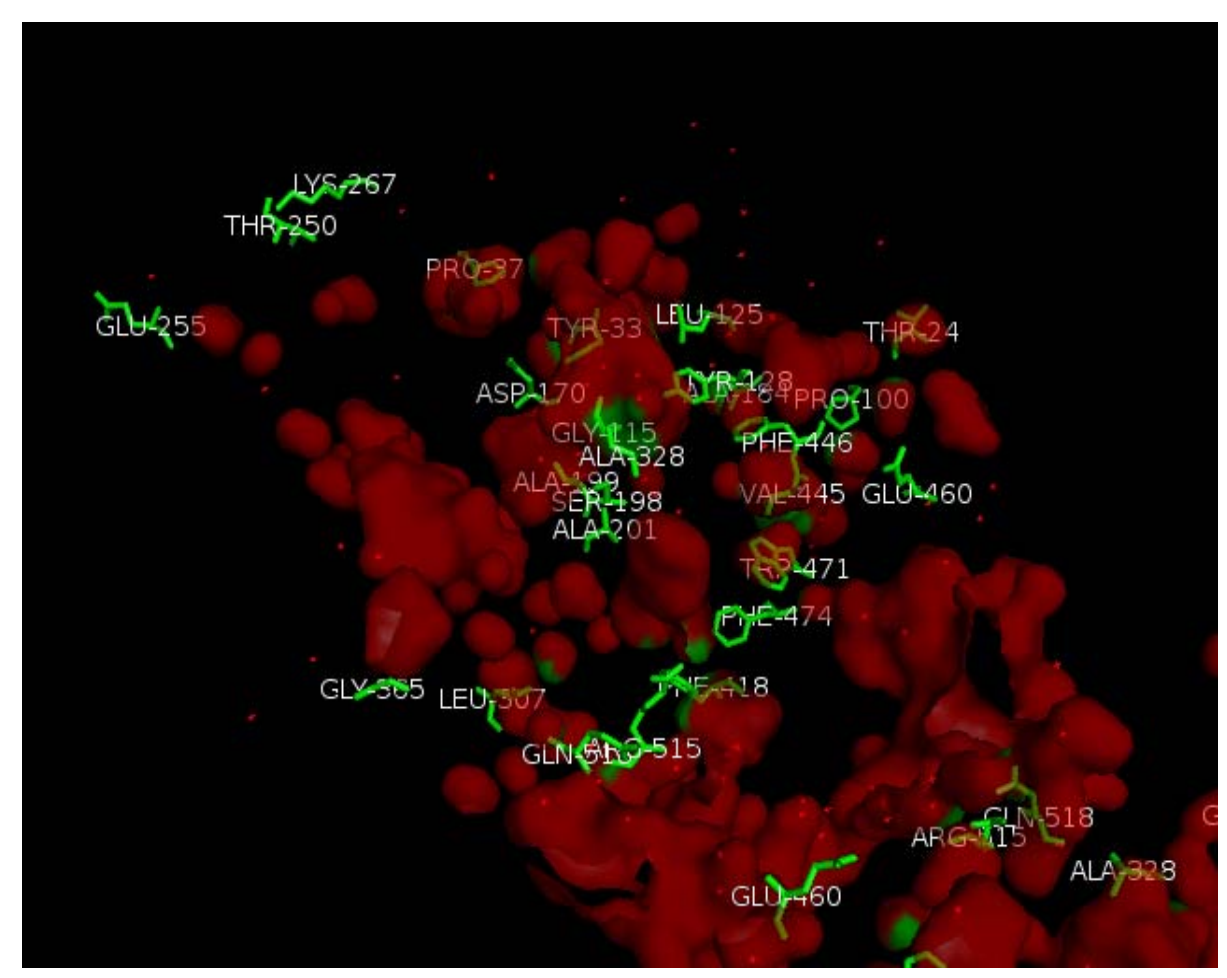

Figure 1. Surface of the Central Cavity of the Active site of experimentally determined hBChE.

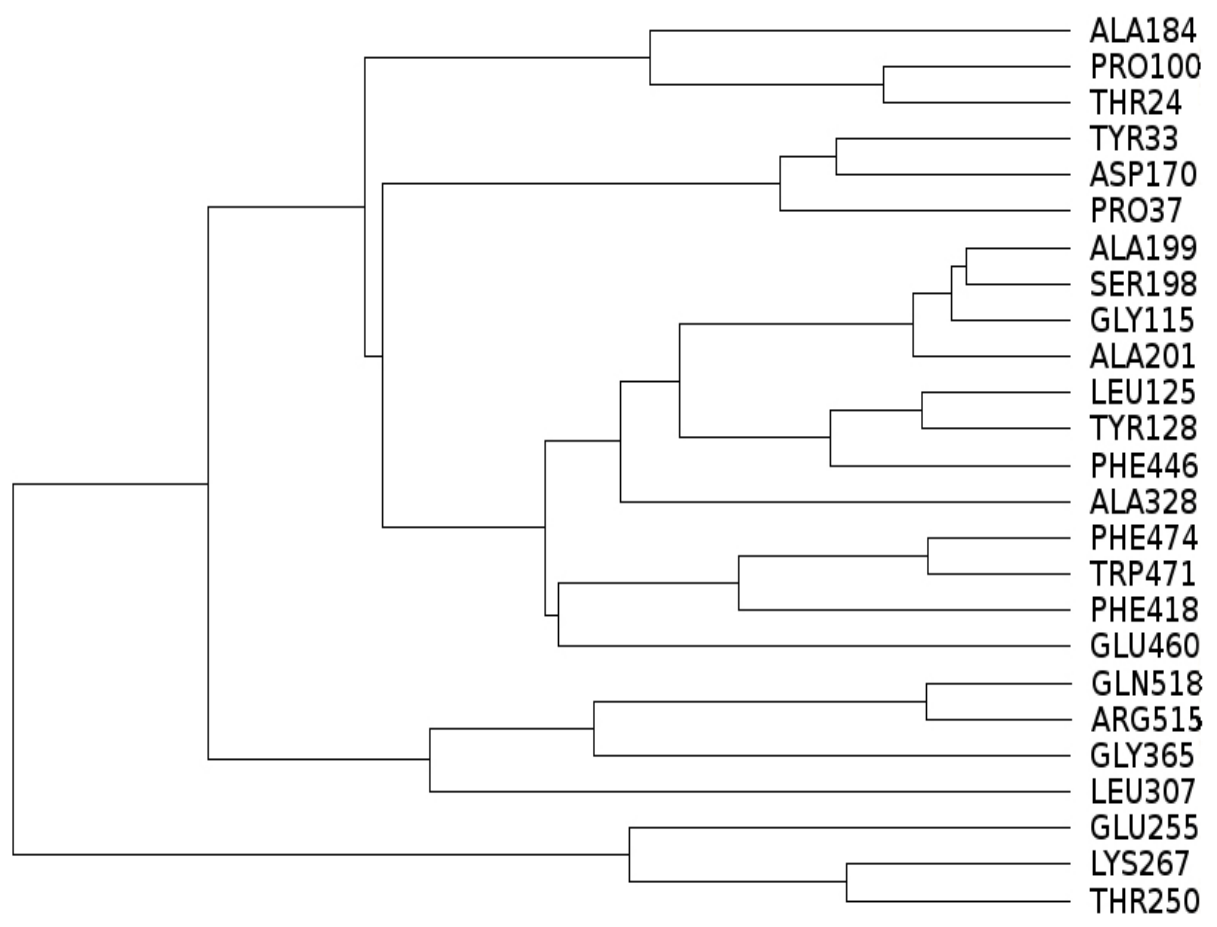

Figure 2. Results of cluster analysis of inter-residue distances for residues whose mutation leads to silent phenoltypes.

S198, A199, G115, A201, L125, Y128, F446, A328. Mutations at these sites would be expected to alter the geometry of the binding site and hence lead to loss of activity. Hydrophobic residues Phe474, Trp471 and
Phe418 form a cluster. Similarly, residues Tyr33, Asp170 and Pro37 form another cluster. The clusters that include the following residues are quite distant from the binding pocket and can be regarded as outliers: Glu255, Lys267, 
Thr250, Leu307, Gly365, Arg515, Gln518. In addition, the following set of residues are also quite distant from the active site: Ala184, Pro100, Thr24, Glu460.

\subsection{Predicted Thermal Stability of Silent Phenotypes}

Loss of activity may be either due to altered geometry of the binding site, or due to loss of structural stability which may lead to denaturation. The thermal stability of the silent phenotypes was predicted to assess the possible contribution of thermodynamic stability. The predicted changes in the free energy of unfolding are presented in Table 2. All silent variants were predicted to have lower thermal stability than the native $\mathrm{BChE}$ based on prediction using Popmusic. Similar results were obtained with I-Mutant2.0, with the following exceptions: Glu255, Gly365 and Gln518. These three variants are predicted to be slightly more stable. However, the predicted increase in stability for these three variants is less than the standard error of the prediction for I-Mutant2.0.

Table 2. Predicted thermal stability and structural perturbations in the variants of human butyryl cholinesterase.

\begin{tabular}{|c|c|c|c|}
\hline $\begin{array}{c}\text { Silent } \\
\text { Variant }\end{array}$ & $\begin{array}{l}\text { Predicted change in thermal } \\
\text { stability by using I-Mutant } 2.0 \\
\text { (kcal/mol) }\end{array}$ & $\begin{array}{l}\text { Predicted change in thermal } \\
\text { stability by using Popmusic } \\
\text { (kcal/mol) }\end{array}$ & $\begin{array}{l}\text { Predicted structural perturbation } \\
\text { at active site by using Modeller } \\
\text { dRMS (A) }\end{array}$ \\
\hline $\mathrm{T} 24 \mathrm{M}$ & -0.03 & -0.7 & 0.39 \\
\hline Y33C & -1.11 & -2.45 & 0.42 \\
\hline P37S & -2.67 & -1.99 & 0.28 \\
\hline P100S & -1.75 & -1.3 & 0.29 \\
\hline G115D & -1.04 & 1.82 & 0.41 \\
\hline $\mathrm{L} 125 \mathrm{~F}$ & -0.07 & -1.26 & 0.31 \\
\hline $\mathrm{Y} 128 \mathrm{C}$ & -1.1 & -2.5 & 0.42 \\
\hline D170A & -1.04 & -0.72 & 0.45 \\
\hline $\mathrm{A} 184 \mathrm{~V}$ & -1.08 & -1.07 & 0.3 \\
\hline S198G & -0.4 & -0.39 & 0.29 \\
\hline A199V & -0.02 & -0.76 & 0.29 \\
\hline A201T & -0.58 & -1.28 & 0.32 \\
\hline $\mathrm{T} 250 \mathrm{P}$ & -2.06 & -2.58 & 0.41 \\
\hline E255D & 0.28 & -0.13 & 0.36 \\
\hline K267R & -1.55 & -0.17 & 0.3 \\
\hline L307P & -1.84 & -2.27 & 0.36 \\
\hline A328D & -0.42 & -1.14 & 0.33 \\
\hline G365R & 0.09 & -0.9 & 0.32 \\
\hline F418S & -2.81 & -4.14 & 0.37 \\
\hline $\mathrm{F} 446 \mathrm{~V}$ & -1.85 & -2.13 & 0.41 \\
\hline $\mathrm{E} 460 \mathrm{~K}$ & -2.59 & -1.29 & 0.39 \\
\hline W471R & -2.83 & -2.68 & 0.41 \\
\hline $\mathrm{F} 474 \mathrm{~L}$ & -1.2 & -1.61 & 0.43 \\
\hline R515C & -0.44 & -0.91 & 0.38 \\
\hline Q518L & 0.81 & -0.04 & 0.4 \\
\hline
\end{tabular}




\subsection{Prediction of Structural Perturbation in the Active Site of Silent Phenotypes}

Models of the structures of silent variants were constructed to assess the possible loss of activity due to altered geometry of the binding site. For each variant, four models were constructed. The models were assessed by using the GA341 scoring function and were found to have a score of 1.0 indicating a good quality of the structure. In addition, the calculated values of the molecular probability density function for all models were large and positive $\left(>19.7 \times 10^{\wedge} 3\right)$, indicating good quality structures. The models were optimized by using conjugate gradients and molecular dynamics. For each variant, the average value of the dRMS for the four models was calculated and used as a quantitative measure of the structural perturbation at the active site. The average value of the dRMS for the four structural models of each variant is listed in Table 2. These results indicate that structural perturbations can be induced in the active site, even by mutations of residues that are quite far from the active site. The calculated value of the dRMS is quite small for all variants, with a maximum of $0.45 \mathrm{~A} \mathrm{ob}-$ served for D170A mutant. However, even small changes in the active site geometry, characterized here by changes in distances between pairs of atoms, can give rise to large effects on the rate of an enzyme catalyzed reaction.

\section{DISCUSSION}

The structural basis for the function of $\mathrm{BChE}$ has been studied by using a number of substrate analogs [6,31]. However, the role of most of the residues observed in the human variants that lack activity have not been elucidated in the earlier structure function studies. The distance based clustering method described here, provides a rapid means for identifying residues that are proximal to residues that are directly involved in the catalytic process (or residues with known interactions with the substrate). Some residues observed in human variants that lack activity were observed in clusters substantially distant from the cluster that includes the residues such as Ser198 that play a role in catalysis; the thermodynamic stability of such residues was assessed in an attempt to explain the observed loss of activity of these variants. Ser198 is known to play a critical role in the catalytic activity of hBChE. The observed silent phenotypes for Ala199, Gly115, Leu125, Tyr128 and Ala328 can be explained based on their proximity to the cavity that defines the binding site based on output from CASTp. Ala201 is also in close proximity to the central cavity that defines the binding site (See Figure 2). The cluster of hydrophobic residues Phe446, Trp471, Phe474, Phe418 is part of a compact core that is likely to be important for maintaining structural integrity (Figure 3). Prediction of thermal

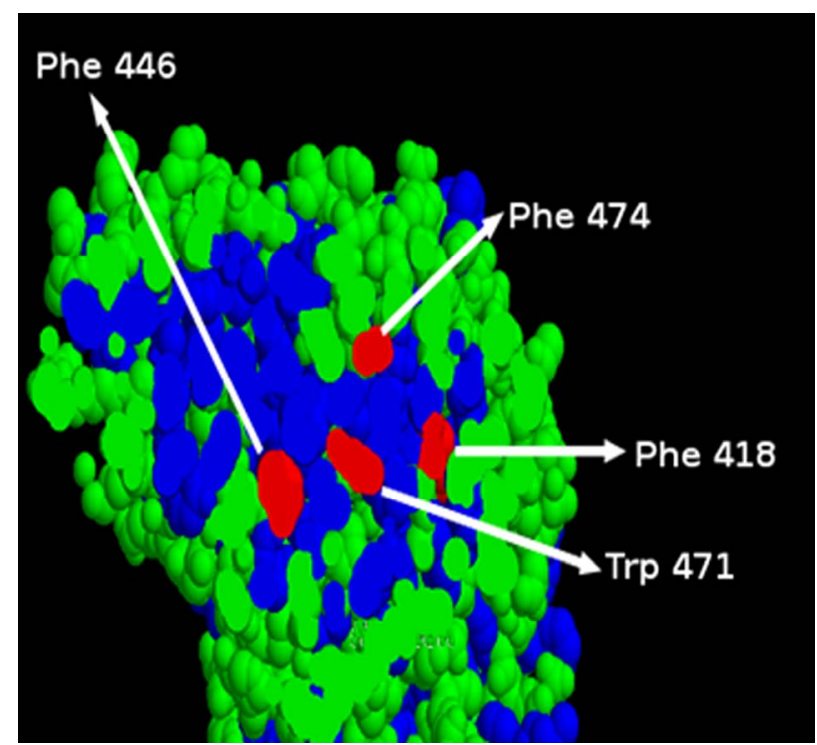

Figure 3. A section of the space-filling model of hBChE.

stability of variants involving these hydrophobic residues indicate these variants are expected to be unstable (Table 2). In addition the prediction of thermal stability indicates that Glu460 has a role in maintaining the structural integrity of $\mathrm{BChE}$.

Leu307 is near the cavity that defines the binding site for MES. This cavity is part of the interface between the two chains in the structure of the full length hBChE. In addition, Leu307 it is mostly buried and mutations may alter the packing and this may lead to changes in positions of residues at the active site. The prediction of thermal stability (Table 2) indicates that this variant will be unstable. The role of Leu307, deduced here, is consistent with earlier predictions [11]. Residues Tyr33 and Thr24 are quite distant from the active site as well as from the regions predicted to play a role in oligomerization. The loss of activity in the T24M mutant does not have an obvious explanation based on the predictions of thermal stability. However, it may induce structural perturbation at the active site as indicated by its dRMS value of 0.39A. The interaction of Tyr33 with Asp170 may be responsible for maintaining long range interactions. Predictions of thermal stability indicate that Y33C will be less stable than the native enzyme. Asp170 is present in the middle of a helix that interacts with thehelix at whose end the active site residue Ser198 is present. Therefore alteration of Asp170 can lead to a minor displacement of the active site residue Ser198, leading to loss of activity. This would explain the observation that, although the predictions of thermal stability indicate only a marginal decrease in thermal stability for the Asp170 variant, the residue Asp170 is highly conserved and its mutation leads to complete loss of activity. The modeling and molecular dynamics calculations also indicate that 
the pair of residues Tyr33 and Asp170 have a higher dRMS $(0.42 \mathrm{~A}$ and $0.45 \mathrm{~A})$ than the average observed for other mutations. A184 is also present at the end of the helix containing Asp170 and the loss of activity in the A $184 \mathrm{~V}$ variant also may be attributed to the same mechanism; however, this is less plausible because A184 is at the end of the helix.

The proximity of Arg515 and Gln518 to the dimerization interface suggests that these residues may play a role in oligomerization. Oligomerization of $\mathrm{BChE}$ is known to play an important role in the turnover of $\mathrm{BChE}$ in serum. However, these two residues are not conserved in $\mathrm{BChE}$ from other species. Gly365 is at the end of a short helix. It is quite far from the cavity defining the active site and is close to the surface. Asanuma et al. [50] have modeled the structure of $\mathrm{hBChE}$ based on the structure of $\mathrm{AChE}$ cholinesterase and based on this model they have suggested the role of steric effects in reduced activity of G365R and other mutants. Prediction of thermal stability of the mutant G365R does not indicate that this mutation will be strongly destabilizing. However, the model for tetramer based on molecular dynamics calculations [51] indicates that this residue is in a helix which has close contacts with another monomeric unit. Therefore, the loss of activity in the G365R mutant can be explained by the inability of the mutant to adopt the native oligomeric form. Mutation of Thr250 to Pro produces a silent phenotype, which can be explained based on the predicted loss of thermal stability (Table 2). However, Thr250 is not conserved in BChE of other species. Lys 267 occurs in a loop on the surface of $\mathrm{BChE}$. This residue is far from the active site. It is spatially close to the terminus of a helix and may play a role in its stabilization. Also, the amino group of lysine side chain may form hydrogen bonds to some neighboring residues, such as Thr250. The prediction of thermal stability indicates that this mutation can be slightly destabilizing. However, its dRMS value $(0.41 \mathrm{~A})$ is higher than average, indicating a possible role in long range perturbation of the active site geometry. $\mathrm{K} 267 \mathrm{R}$ is listed as a silent mutation in the Esther database. However, examination of the data in the paper cited in Esther [7] indicates the possibility that the mutation of interest, K267R, may have low activity. Glu255 occurs in a loop on the surface of $\mathrm{BChE}$ and its side chain points away from the rest of the protein. Glu255 is not conserved in $\mathrm{BChE}$ sequences of other species. In addition, the predictions of thermal stability (Table 2) indicate this mutation would have little or no effect on the thermal stability of the monomer. The computed model of the tetramer of BChE indicates that Glu255, Thr250 and Lys 267 are in close proximity to another monomer in the tetramer. This indicates a possible role for these three residues in the stabilization of the tetramer. Failure to form a tetramer may lead to rapid clearance of the monomeric forms from the serum, even if they retain catalytic activity. However, it is known that $\mathrm{BChE}$ can form oligomers with a structure that is substantially different from the physiologically relevant oligomers [32]. The current computational methods cannot give quantitative predictions regarding the stability of variants of these oligomers with adequate precision. Therefore, the conclusions regarding the roles of residues implicated in oligomerization are only of a qualitative nature.

The variants of human $\mathrm{BChE}$ that lead to loss of activity were examined for structural changes near the known active site and the thermodynamic stability of the variants was estimated. These studies indicate that the observed loss of activity of the variants can, in most cases, be attributed to structural changes near the active site, and/or to decrease in thermodynamic stability. Our findings are consistent with a recent report by Vyas et al. [52], who demonstrated the effect of mutations near the active site using molecular dynamics simulations. Similarly, molecular modeling of the TS1 structures showed that mutations on certain nonactive site residues can indirectly affect the catalytic efficiency of the enzyme against (-)-cocaine through enhancement or weakening of the overall hydrogen bonding between the carbonyl oxygen of (-)-cocaine benzoyl ester and the oxyanion hole of the enzyme [53].

The underlying theme of our study was to assess alterations in structure and thermodynamic stability of $\mathrm{BChE}$ variants, because modulation of cholinergic nervous system is useful to understand the pathogenesis of Alzheimer's disease and to drugs that modify the symptoms and possibly its course. Bartels et al. have demonstrated that the stability of the K-variant of $\mathrm{BChE}$ (A539T) remains unchanged and that its (succinyl cholinesterase) activity is only slightly lower than that of native $\mathrm{BChE}$, indicating only minor structural changes at the active site [54]. Hence, the association of the K-variant of $\mathrm{BChE}$ with Alzheimer's disease is likely due to altered intermolecular interactions [54].

A knock-out model of $\mathrm{BChE}$ has been developed that mimics many metabolic changes of variant $\mathrm{BChE}$ properties in humans such as hydrolysis of toxins and involvement in obesity $[55,56]$. However there are differences in the animal models and humans such as the need for double knock out (viz both BChE and carboxylesterase) [57]. In addition, preliminary studies in subjects with BChE variants have not shown any adverse health effects, other than an inability to rapidly metabolize succinylcholine, a muscle relaxant used in anesthetic practice [58]. However, further prospective studies can be performed in subjects with lack of BChE activity [25] to evaluate the cognitive status associated with aging, which can provide insights into the possible role of $\mathrm{BChE}$ in influencing cortical and higher functions. Such information 
can be useful in designing biomarkers or therapeutic agents for the early diagnosis and treatment of cognitive dysfunction associated with aging.

\section{REFERENCES}

[1] Orhan, I.E. (2012) Current concepts on selected plant secondary metabolites with promising inhibitory effects against enzymes linked to Alzheimer's disease. Current Medicinal Chemistry, 19, 2252-2261.

[2] Pohanka, M. (2011) Cholinesterases, a target of pharmacology and toxicology. Biomedical Papers of the Medical Faculty of the University Palacký, 155, 212-229. doi:10.5507/bp.2011.036

[3] Goodall, R. (2004) Cholinesterase: Phenotyping and genotyping. Annals of Clinical Biochemistry, 41, 98-110. doi:10.1258/000456304322879971

[4] Taylor, P. (1991) The cholinesterases. Journal of Biological Chemistry, 266, 4025-4028.

[5] Silman, I. and Sussman, J.L. (2008) Acetylcholinesterase: How is structure related to function? Chemico-Biological Interactions, 175, 3-10. doi:10.1016/j.cbi.2008.05.035

[6] Cokugras, A.N. (2003) Butyrylcholinesterase: Structure and physiological importance. Turkish Journal of Biochemistry, 28, 54-61.

[7] Maekawa, M., Sudo, K., Dey, D.C., Ishikawa, J., Izumi, M., Kotani. K. and Kanno, T. (1997) Genetic mutations of butyrylcholine esterase identified from phenotypic abnormalities in Japan. Clinical Chemistry, 43, 924-929.

[8] Levano, S., Ginz, H., Siegemund, M., Flipovic, M., Voronkov, E., Urwyler, A. and Girard, T. (2005) Genotyping the butyrylcholinesterase in patients with prolonged neuromuscular block after succinylcholine. Anesthesiology, 102, 531-535.

[9] Parmo, S.L.P., Bartels, C.F., Wiersema, B., van der Spek, A.F., Innis, J.W. and La Du, B.N. (1996) Characterization of 12 silent alleles of the human butyrylcholinesterase (BCHE) gene. American Journal of Human Genetics, 58, 52-64.

[10] Boberg, D.R., Alle, L.F., Soua, L.R. and Maia, E.A.C. (2010) Molecular forms of butrylcholinesterase and obesity. Genetics and Molecular Biology, 33, 452-454. doi:10.1590/S1415-47572010005000072

[11] Manoharan, I., Wieseler, S., Layer, P.G., Lockridge, O. and Boopathy, R. (2006) Naturally occurring mutation Leu307Pro of human butyrylcholinesterase in the Vysya community of India. Pharmacogenetics and Genomics, 16, 461-468.

[12] Ciro, A., Park, J., Burkhard, G., Yan, N. and Geula, C. (2012) Biochemical differentiation of cholinesterases from normal and Alzheimer's disease cortex. Current Alzheimer Research, 9, 138-143. doi:10.2174/156720512799015127

[13] Garcia-Ayllon, M.S., Riba-Liena, I., Serra-Basante, C., Alom, J., Boopathy, R. and Saez-Valero, J. (2010) Altered levels of acetylcholinesterase in Alzheimer plasma. PLoS One, 5, e8701.

[14] Darvesh, S., Reid, G.A. and Martin, E. (2010) Biochemi- cal and histochemical comparison of cholinesterases in normal and Alzheimer brain tissues. Current Alzheimer Research, 7, 386-400. doi:10.2174/156720510791383868

[15] Podoly, E., Hanin, G. and Soreq, H. (2010) Alanine-tothreonine substitutions and amyloid diseases: Butyrylcholinesterase as a case-study. Chemico-Biological Interactions, 187, 64-71. doi:10.1016/j.cbi.2010.01.003

[16] Bullock, R. and Lane, R. (2007) Executive dyscontrol in dementia, with emhasis on subcortical pathology and the role of butyrylcholinesterase. Current Alzheimer Research, 4, 277-293. doi:10.2174/156720507781077313

[17] Darreh-Shori, T., Forsberg, A., Modiri, N., Andreasen, N., Blennow, K., Kamil, C., Ahmed, H., Almkvist, O., Langstrom, B. and Nordberg, A. (2011) Differential levels of apolipoprotein $\mathrm{E}$ and butyrylcholinesterase show strong association with pathological signs of Alzheimer's disease in the brain in vivo. Neurobiology of Aging, 32, e15e32.

[18] Lane, R.M. and He, Y. (2012) Butyrylcholinesterase genotype and gender influence Alzheimer's disease phenotype. Alzheimers Dement, in press. doi:10.1016/j.jalz.2010.12.005

[19] Darreh-Shori, T., Siawesh, M., Mousavi, M., Andreasen, N. and Nodberg, A. (2012) Apoliporotein E4 modulates phenotype of butyrylcholinesterase in CSF of patients with Alzheime's disease. Journal of Alzheimers Disease, 28, 443-458.

[20] Darreh-Shori, T., Modiri, N., Blennow, K., Baza, S., Kamil, C., Ahmed, H., Andreasen, N. and Nordberg, A. (2011) The apolipoprotein E $\varepsilon 4$ allele plays pathological roles in $\mathrm{AD}$ through high protein expression and interacttion with butyrylcholinesterase. Neurobiology of Aging, 32, 12461248. doi:10.1016/j.neurobiolaging.2009.07.015

[21] Lane, R., Feldman, H.H., Meyer, J., He, Y., Ferris, S.H., Nordberg, A., Darreh-Shori, T., Soininen, H., Pirttila, T., Farlow, M.R., Sfikas, N., Ballard, C. and Greig, N.H. (2008) Synergistic effect of apolipoprotein E epsilon4 and butyrylcholinesterase K-variant on progression from mild cognitive impairment to Alzheimer's disease. Pharmacogenet Genomics, 18, 289-298. doi:10.1097/FPC.0b013e3282f63f29

[22] Lane, R., He, Y., Morris, C., Leverenz, J.B., Emre, M. and Ballard, C. (2009) BuChe-K and APOE epsilon4 allele frequencies in Lewy body dementias, and influence of genotype and hyperhomocysteinemia on cognitive decline. Movement Disorders, 24, 392-400. doi: $10.1002 / \mathrm{mds} .22357$

[23] Mateo, I., Llorca, J., Infante, J., Rodriguez-Rodriguez, E., Berciano, J. and Combarros, O. (2008) Gene-gene interaction between 14-3-3 zeta and butyrylcholinesterase modulates Alzheimer's disease risk. European Journal of Neurology, 15, 219-222. doi:10.1111/j.1468-1331.2008.02059.x

[24] Sridhar, G.R., Rao, A.A., Srinivas, K., Nirmala, G., Lakshmi, G., et al. (2010) Butyrylcholinesterase in metabolic syndrome. Medical Hypotheses, 75, 648-651. doi:10.1016/j.mehy.2010.08.008

[25] Sridhar, G.R., Thota, H., Allam, A.R., et al. (2006) Alzheimer's disease and type 2 diabetes mellitus: The choli- 
nesterase connection? Lipids in Health and Disease, 5, 28. doi:10.1186/1476-511X-5-28

[26] Sridhar, G.R. and Nirmala, G. (2002) Inborn errors in lipid metabolism. In: Tripathy, B.B. and Das, S. Eds., Lipid Disorders, Association of Physicians of India, College of Physicians, Guwahati, 59-80.

[27] Sridhar, G.R. (2011) Proteins of the esterase family: Patents for some proteins in search of metabolic functions. Recent Patents on Biomarkers, 1, 205-212.

[28] Deniz-Naranjo, M.C., Munoz-Fernande, C., Alemany-Rodriguez, M.J., del Perez-Vieitez, C.M., Aldro-Benito, Y., Irurita-Latasa, J. and Sanchez-Gracia, F. (2007) Butyrylcholinesterase, ApoE and Alzheimer's disease in a population from the Canary Islands (Spain). Neuroscience Letters, 427, 34-38. doi:10.1016/j.neulet.2007.08.059

[29] Piccardi, M., Congiu, D., Squassina, A., Manconi, F., Putzu, P.F., Mereu, R.M., Chillotti, C. and Del Zompo, M. (2007) Alzheimer's disease: Case-control association study of polymorphisms in ACHE, CHAT, and BCHE genes in a Sardinian sample. American Journal of Medical Genetics Part B: Neuropsychiatric Genetics, 144B, 895-899. doi:10.1002/ajmg.b.30548

[30] Luschchekina, S.V., Grigorenko, B.L., Morozov, D.I., Polyakov, I.V., Nemukhin, A.V. and Varfolomeev, S.D. (2010) Modeling of the mechanism of hydrolysis of succinylcholine in the active site of native and modified (Asp70Gly) human butyrylcholinesterase. Russian Chemical Bulletin, 59, 55-60. doi:10.1007/s11172-010-0044-0

[31] Nicolet, Y., Lockridge, O., Masson, P., Fontecilla-camps, J.C. and Nachon, F. (2003) Crystal structure of human butyrylcholinesterase and of its complexes with substrate and products. Journal of Biological Chemistry, 278, 4114141147. doi:10.1074/jbc.M210241200

[32] Ngamelue, M.N., Homma, K., Lockridge, O. and Asojo, O.A. (2007) Crystallization and X-Ray structure of full length recombinant human butyrylcholinesterase. Acta Crystallographica Section F: Structural Biology and Crystallization Communications, 63, 723-727. doi:10.1107/S1744309107037335

[33] Worth, C.L., Bickerton, G.R., Schreyer, A., Forman, J.R., Cheng, T.M., Lee, S., Gong, S., Burke, D.F. and Blundell, T.L. (2007) A structural bioinformatics approach to the analysis of nonsynonymous single nucleotide polymorphisms (nsSNPs) and their relation to disease. Journal of Bioinformatics and Computational Biology, 5, 1297-1318. doi:10.1142/S0219720007003120

[34] Hotelier, T., Renault, L., Cousin, X., Negre, V., Marchot, P. and Chatonnet, A. (2004) ESTHER, the database of the $\alpha / \beta$-hydrolase fold superfamily of proteins. Nucleic Acids Research, 32, D145-D147. doi:10.1093/nar/gkh141

[35] Corpet, F. (1988) Multiple sequence alignment with hierarchical clustering. Nucleic Acids Research, 16, 1088110890. doi:10.1093/nar/16.22.10881

[36] Thompson, J.D., Higgins, D.G. and Gibson, T.J. (1994) CLUSTAL W: Improving the sensitivity of progressive multiple sequence alignment through sequence weighting, position-specific gap penalties and weight matrix choice. Nucleic Acids Research, 22, 4673-4680. doi:10.1093/nar/22.22.4673
[37] Bernstein, F.C., Koetzle, T.F., Williams, G.J.B., Meyer, E.F., Brice, M.D. Jr., Rodgers, J.R., Kennard, O., Shimanouchi, T. and Tasumi, M. (1977) The protein databank: A computer-based archival file for macromolecular structures. Journal of Molecular Biology, 112, 535-542. doi:10.1016/S0022-2836(77)80200-3

[38] Berman, H.M., Westbrook, J., Feng, Z., Gilliland, G., Bhat, T.N., Weissig, H., Shindyalov, I.N. and Bourne, P.E. (2000) The Protein Data Bank. Nucleic Acids Research, 28, 235242. doi:10.1093/nar/28.1.235

[39] Carletti, E., Li, H., Li, B., Ekstrom, F., Nicolet, Y., Loiodice, M., Gillon, E., Froment, M.T., Lockridge, O., Schopfer, L.M., Masson, P. and Nachon, F. (2008) Aging of cholinesterases phosphorylated by tabun proceeds through odealkylation. Journal of the American Chemical Society, 130, 16011-16020. doi:10.1021/ja804941z

[40] Felsenstein, J. (1989) PHYLIP: Phylogeny Inference Package (Version 3.2). University of Washington, Seattle.

[41] Treeview. http://darwin.zoology.gla.ac.uk/ rpage/treeviewx/index.ht $\underline{\mathrm{ml}}$

[42] Perrière, G. and Gouy, M. (1996) WWW-query: An on-line retrieval system for biological sequence banks. Biochimie, 78, 364-369. doi:10.1016/000-9084(96)84768-7

[43] De Lano, W. The PyMOL Molecular Graphics System, Schrödinger, LLC. http://www.pymol.org

[44] Liang, J., Edelsbrunner, H. and Woodward, C. (1998) Anatomy of protein pockets and cavities: Measurement of binding site geometry and implications for ligand design. Protein Science, 7, 1884-1897. doi:10.1002/pro.5560070905

[45] Dundas, J., Ouyang, Z., Tseng, J., Binkowski, A., Turpaz, Y. and Liang, J. (2006) CASTp: Computed atlas of surface topography of proteins with structural and topographical mapping of functionally annotated residues. $\mathrm{Nu}$ cleic Acids Research, 34, W116-W118. doi:10.1093/nar/gk1282

[46] Dehouck, Y., Grosfils, A., Folch, B., Gilis, D., Bogaerts, P. and Rooman, M. (2009) Fast and accurate predictions of protein stability changes upon mutations using statistical potentials and neural networks: PoPMuSiC-2.0. Bioinformatics, 25, 2537-2543. doi:10.1093/bioinformatics/btp445

[47] Capriotti, E., Fariselli, P. and Casadi, R. (2005) I-Mutant2.0: Predicting stability changes upon mutation from the protein sequence or structure. Nucleic Acids Research, 33, W306-W310. doi:10.1093/nar/gki375

[48] Sali, A. and Blundell, T.L. (1993) Comparative protein modelling by satisfaction of spatial restraints. Journal of Molecular Biology, 234, 779-815. doi:10.1006/jmbi.1993.1626

[49] Marti-Renom, M.A., Stuart, A., Fiser, A., Sánchez, R., Melo, F. and Sali, A. (2000) Comparative protein structure modeling of genes and genomes. Annual Review of Biophysics and Biomolecular Structure, 29, 291-325.

[50] Asanuma, K., Yagihashi, A., Yagihashi, A., Uehara, N., Kida, T. and Watanabe, N. (1999) Three point mutations 
of human butyrylcholinesterase in a Japanese family and the alterations of three-dimensional structure. Clinica Chimica Acta, 283, 33-42. doi:10.1016/S0009-8981(99)00030-3

[51] Pan, Y., Muzyka, J. and Zhan, C.G. (2009) Model of human butyrylcholinesterase (BChE) tetramer by homology modeling and dynamics simulation. Journal of Physical Chemistry B, 113, 6543-6552. doi:10.1021/jp8114995

[52] Vyas, S., Beck, J.M., Xia, S., Zhang, J. and Hadad, C.M. (2010) Butyrylcholinesterase and G116H, G116S, G117H, G117N, E197Q and G117H/E197Q mutants: A molecular dynamics study. Chemico-Biological Interactions, 187, 241-245. doi:10.1016/j.cbi.2010.04.004

[53] Zheng, F., Yang, W., Xue, L., Hou, S., Liu, J. and Zhan, C.G. (2010) Design of high-activity mutants of human butyrylcholinesterase against (-)-cocaine: Structural and energetic factors affecting the catalytic efficiency. Biochemistry, 49, 9113-9119. doi:10.1021/bi1011628

[54] Bartels, C.F., Jensen, F.S., Lockridge, O., van der Spek A, F.L., Rubinstein, H.M., Lubrano, T., La Du, B.N. (1992) DNA mutation associated with the human butyrylcholinsterase K-variant and its linkage to the atypical variant mutation and other polymorphic sites. American Journal of Human Genetics, 50, 1086-1103.

[55] Li, B., Duysen, E.G., Carlson, M. and Lockridge, O. (2008) The butyrylcholinesterase knockout mouse as a model for human butyrylcholinesterase deficiency. Journal of Pharmacology and Experimental Therapeutics, 324, 1146-1154. doi:10.1124/jpet.107.133330

[56] Li, B., Duysen, E.G. and Lockridge, O. (2008) The butyrylcholinesterase knockout mouse is obese on a high-fat diet. Chemico-Biological Interactions, 175, 88-91. doi:10.1016/j.cbi.2008.03.009

[57] Duysen, E.G., Li, B. and Lockridge, O. (2009). The butyrylcholinesterase knockout mouse as a research tool in the study of drug sensitivity, bio-distribution, obesity and Alzheimer's disease. Expert Opinion on Drug Metabolism and Toxicology, 5, 523-528.

[58] Indumathi, M., Rathnam, B., Sultan, D. and Lockridge, O. (2007) A medical health report on individuals with silent butyrylcholinesterase in the Vysya community of India. Clinica Chimica Acta, 378, 128-135. doi:10.1016/j.cca.2006.11.005 